\title{
Stability of the human spine: a biomechanical study
}

\author{
P J M Scholten* PhD \\ A G Veldhuizen* MD and H J Grootenboer** PhD
}

* Department of Orthopaedics, State University, Groningen, The Netherlands

* Department of Mechanics, Twente University of Technology, Enschede, The Netherlands

\begin{abstract}
Summary
The influences of curvatures and of physical properties on the mechanical stability of the spine were analysed by means of a three-dimensional, geometrical, nonlinear biomechanical model. According to the model, the initial buckling load decreases with increasing lordotic and kyphotic curvatures. When the body weight is taken into account as a load distributed along the whole spine, the calculated initial buckling load is twice the value that it is in the case of a single concentrated load acting at the top of the spine. Applying the large deflection theory, no relation is found between the increased slenderness of a spine and a 'buckled' configuration of a scoliotic spine.
\end{abstract}

\section{Relevance}

The literature consistently supports the hypothesis that scoliosis can be mediated through buckling phenomena in a purely mechanical manner. This paper shows that idiopathic scoliosis and its progression cannot be explained on the basis of the column buckling theory.

Key words: Stability, Idiopathic scoliosis, Spine, Biomechanics

\section{Introduction}

A number of factors may be responsible for the deformity of the spine in idiopathic scoliosis, such as genetic, biochemical, hormonal, neurological and mechanical factors ${ }^{1}$. From a mechanical point of vicw it is interesting to note that many individuals have slight asymmetries of their pelvis, slight leg length discrepancies, and a slight right thoracic curve ${ }^{2}$. White and Panjabi ${ }^{3}$ suggested that this slight thoracic curvature might result in asymmetrical loading and muscle imbalance. According to White, such an occurrence can generate progression to scoliosis. Others have hypothesized that asymmetric muscle strength is of aetiological importance in the formation of scoliosis ${ }^{4.5}$. A biomechanical model study is helpful in analysing what factor may be a cause of scoliosis rather than a secondary effect. There is an interesting mechanical analogy between the stability in a slender strut and a scoliotic spine. The first problems of elastic stability, concerning lateral buckling of compressed members, were solved about 200 years ago by Euler. Several researchers have used the

Submitted: 7 July 1987 In revised form: 12 September 1987 Correspondence and reprim reculests (o): Dr P J M Scholten. College of Advanced Technology. Oudenoard 7(x). 3513 EX Utrecht. The Netherlands.
Euler theory to explain the mechanical stability of the human spine. As far as we know, Fick ${ }^{6}$ was the first to use this linear elastic theory to explain the buckling behaviour of the human spine. Lucas and Bresler ${ }^{7}$ have calculated by means of the buckling formula of Euler the initial buckling load of the human spine. Haderspeck et al. ${ }^{8}$ reported: 'In biomechanical terms, the progression of scoliosis can be likened to the buckling of an elastic column'. Mattson et al." reported: 'The progression of a lateral spine curve in scoliosis can be thought of in biomechanical terms as a gradual buckling of the flexible spinal column'. Pope et al.' reported that because of the lordotic and kyphotic curvatures of the spine the initiation of a lateral curvature by a buckling mechanism is less likely.

The purpose of this study is to investigate whether the initiation of scoliosis can be explained by means of the buckling theory. In addition, two parameters were analysed: the influence of the curvature of the spine and the influence of the physical properties of the spine on its mechanical stability.

\section{Stability}

Stability can be considered from a clinical, anatomical or mechanical point of view. Mechanical stability is 
different from what is referred to as clinical or anatomical stability. Clinical stability is the ability of the spine, within a range of physiological loading, to limit patterns of displacement and to prevent deformity or pain due to structural changes ${ }^{3}$. Clinical instability is always associated with an abnormal deformation and a loss of tissue stiffness. Anatomical stability is based on morphometric parameters and does not take into account the adaptation of structures. Anatomical stability of a joint can be described as a measure of mobility of that joint. A joint in a closed-packed or locked position is said to be stable, because the compression caused by the surrounding structures results in less mobility. Clinical as well as anatomical stability are more or less qualitative descriptions, in contrast to mechanical stability. Mechanical stability. or a stable position of the spine, can be described as a situation in which there is a consistent relationship between this position and the forces needed to maintain this position. and unstable when a strong change of this position is caused by only a small change in the applied forces. In mechanical terms a sufficient condition for mechanical stability for elastic systems subject to conservative loading is a positive definite second variation of the potential energy about an equilibrium state.

An example of a mechanically unstable situation is shown in Figure 1. A motion segment of the spine without ligaments and with the nucleus pulposus considered as a ball has too many degrees of freedom. It is mechanically unstable. A motion segment with ligaments is mechanically stable, but can be clinically unstable. The human spine can be described as a long slender rod, as shown in Figure 2. Loading such a rod results in a compression of this rod. This is a stable situation. When the compression load, acting at the rod, is too high, a sudden bending or buckling will occur. This is an unstable situation. The load required to reach this unstable situation is called the initial buckling load. This buckling load can be described as the upper limit of loading the spine at which there is a transition between a stable and an unstable situation.

The buckling load $P_{k}$ depends upon the length $L$ of the rod, the manner in which the rod is supported, the

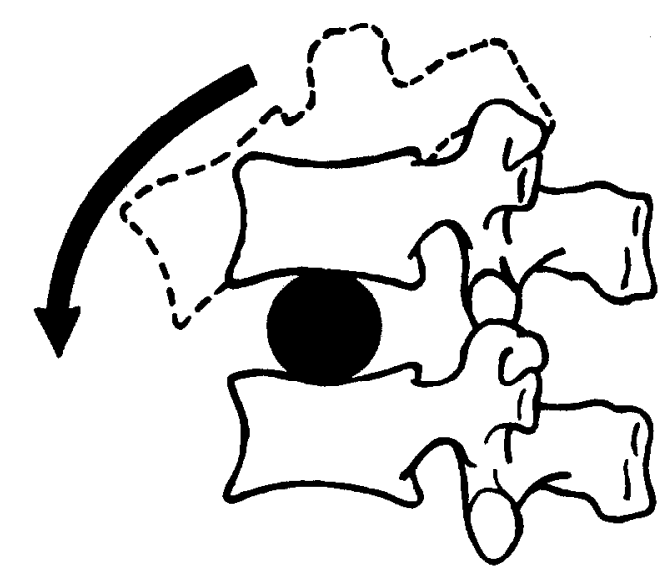

Figure 1. An example of a mechanically unstable system.

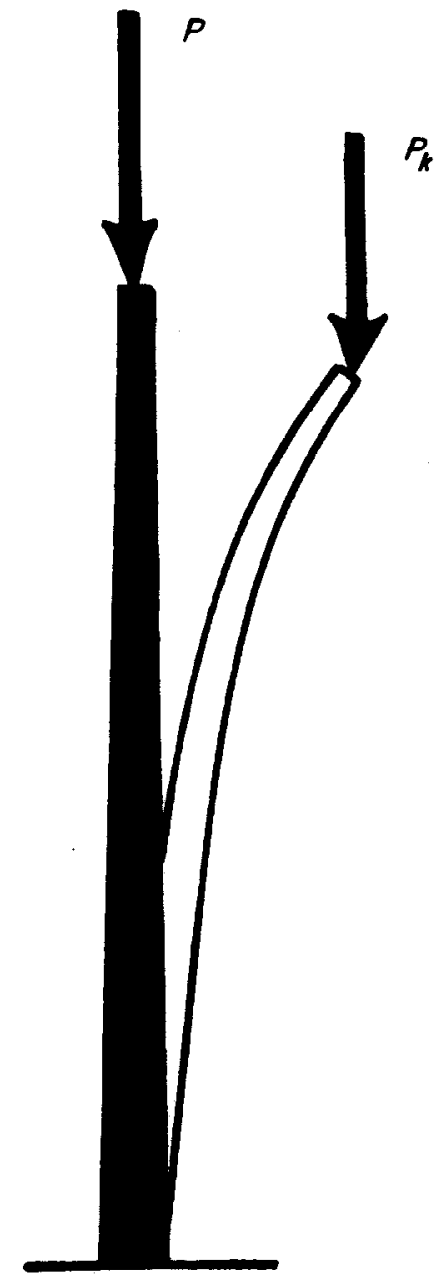

Figure 2. The human spine represented as a long slender rod. There is mechanical stability for $P<P_{k}$, with $P_{k}$ the initial buckling load.

resistance against bending $\mathrm{EI}$ and the force system. There are two force systems acting on the spine. The first system is caused by the weight of the body and the second system is the force system exerted by the muscles. The first system is always working in the vertical direction and is independent of the deflection of the spine. The second system is an example of a nonconservative force system. The position and direction of a muscle force is dependent on the deflection of the spinc. This means that the resulting muscle force on the spine can act in the direction of the tangent to the deflection curve. The buckling load $P_{k}$ in the case of a conservative force acting on a rod fixed at one end and free to move at the other end is expressed by the buckling formula of Euler, and equals:

$$
P_{k}=\frac{\pi^{2} E I}{4 L^{2}}
$$

The buckling load for the same rod as described above with a non-conservative force acting at the top and directed to the fixed end equals:

$$
P_{k}=2 \cdot 008 * \frac{\pi^{2} E I}{L^{2}}\left(\text { Timoshenko }{ }^{10}\right)
$$



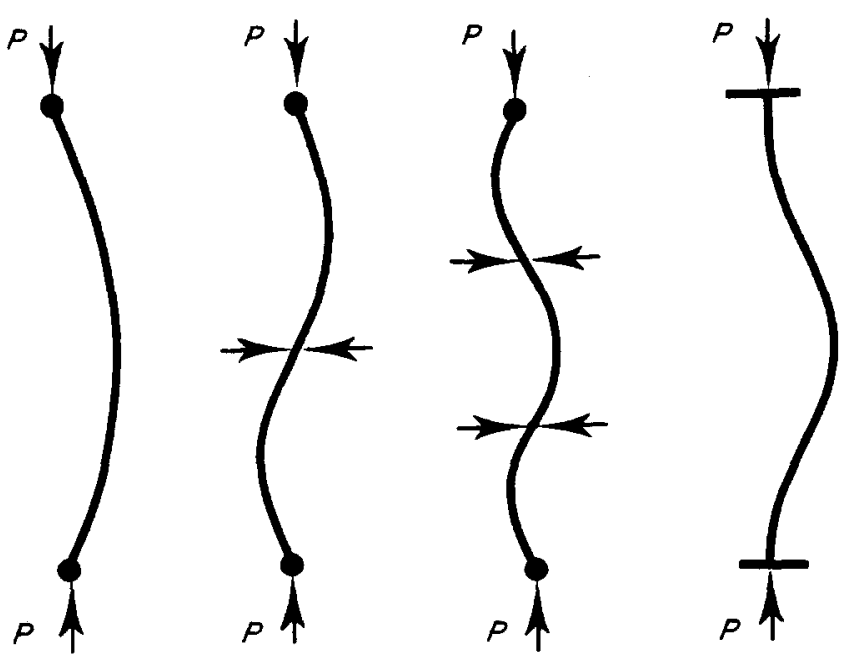

Figure 3. Different modes of buckling.

From this buckling formula, it follows that the maximal compression force the rod can carry is highly dependent upon the length $\mathrm{L}$ of the spine. The buckling load is inversely proportional to the square of the length $\mathrm{L}$ and proportional to the bending stiffness EI. From both formulae it follows that the most critical force system is the conservative force system exerted by the body weight.

In Figure 3 different modes of buckling in slender struts are shown. If scoliosis can be considered as a buckling process, single, double, and multiple curves can be produced for higher buckling loads by applying external lateral constraints at the inflection points of the spine. So, theoretically the rib cage is a system that can prevent buckling of the spine. The end constraints for the spine are normally such that the pelvis and the occiput must remain horizontal and that, in general, the spine is 'in balance', which means that the head is vertically above the pelvis. These end constraints may be the most realistic boundary conditions.

\section{The initial buckling load of the human spine}

The initial buckling load of the human spine can be described as the upper limit of loading the spine with a stable behaviour. For a straight rod, the initial buckling load can be calculated using the buckling formula of Euler. In order to take into account the curvature of the spine, a discrete model of the spine was used by Scholten ${ }^{11}$. This model is a three-dimensional geometrical nonlinear computer model, representing the spine as a collection of 17 rigid bodies, each corresponding to a thoracic or lumbar vertebra, and interconnected by deformable elements, corresponding to the intervertebral discs, the intervertebral joints and three ligaments. Measurements by means of X-rays were made to obtain a geometrical description of the spine configuration. The physical properties were taken from a study by Schultz et al. ${ }^{12}$.
The potential energy PE of the structure of the spine model can be given by:

$$
P E=1 / 2 u^{T} u-u^{T} P
$$

with $u$ : displacement vector

$\mathrm{u}^{\mathrm{T}}$ : transposed vector $\mathrm{u}$

$P$ : load vector

$\mathrm{K}=\mathrm{K}_{\mathbf{0}}+\mathrm{K}$

$K_{(1)}$ : linear stiffness matrix

$\mathrm{K}_{\mathbf{r}}$ : initial stress matrix.

The initial buckling load $P_{k}$ can be calculated by vanishing the second variation $\delta^{2}(\mathrm{PE})$ for arbitrary variations, $\delta u$, of the displacement vector $u$. Using the linear elastic theory, this condition is given by the equation: det $\left(K_{0}+\lambda K_{s}\right)=0$. The initial buckling load $P_{k}$ corresponds with the lowest eigenvalue $\lambda$ of $\operatorname{det}\left(K_{0}+\lambda K_{s}\right)=0$ and $P_{k}=\lambda P$.

The initial buckling load of a normal curved spine was calculated. The sacrum was fixed and the top of the spine model was free to move. The load vector $P$ is a vertical load acting at the top of the thoracic part of the spine. The obtained initial buckling load was found to be $217 \mathrm{~N}$. This value is in agreement with results obtained by Lucas and Bresler ${ }^{7}$ and Belytschko et al: ${ }^{1.3}$.

By taking into account the distribution of the body weight along the spine by constructing the load vector $P$ out of several forces, an initial buckling load was found to be twice as great as in the case of a single vertical load vector $P$ acting at the top of the spine. This difference is easy to explain. Using a single load acting at the top of the spine, the length of the buckling column is equal to the length of the spine. By taking into account the distribution of the body weight as several forces, the length of the buckling column is the length between the bottom of the spine and the point of the resultant force of the body weight. This length is shorter than the length of the spine and therefore will increase the amount of the buckling load.

In order to analyse the influence of the curvature of the spine on the initial buckling load, the abovedescribed minimum potential energy criterion was used. The curvatures in the sagittal plane of both the thoracic and the lumbar parts of the spine were varied. This was done by multiplying the horizontal coordinates and the inclinations of the thoracic vertebrae with a factor $\chi$. This factor was, respectively, $0,0 \cdot 5,1$ and 1.5. The corresponding shapes are called, respectively, straight, flat, normal and prominent. The same variation was performed for the lumbar part of the spine. In this way 16 different shapes were obtained with equal height. In Figure 4 various shapes of the thoracic and lumbar spine are drawn.

The initial buckling loads of the 16 different shapes were calculated and expressed with respect to a straight spine. So, the buckling load of a straight spine is $100 \%$. According to the model, the initial buckling load of the spine decreases with increasing lordotic and kyphotic curvature, see Figure 5. The initial buckling load for a normal curved spine is about $60 \%$ of a spine without curvature. 


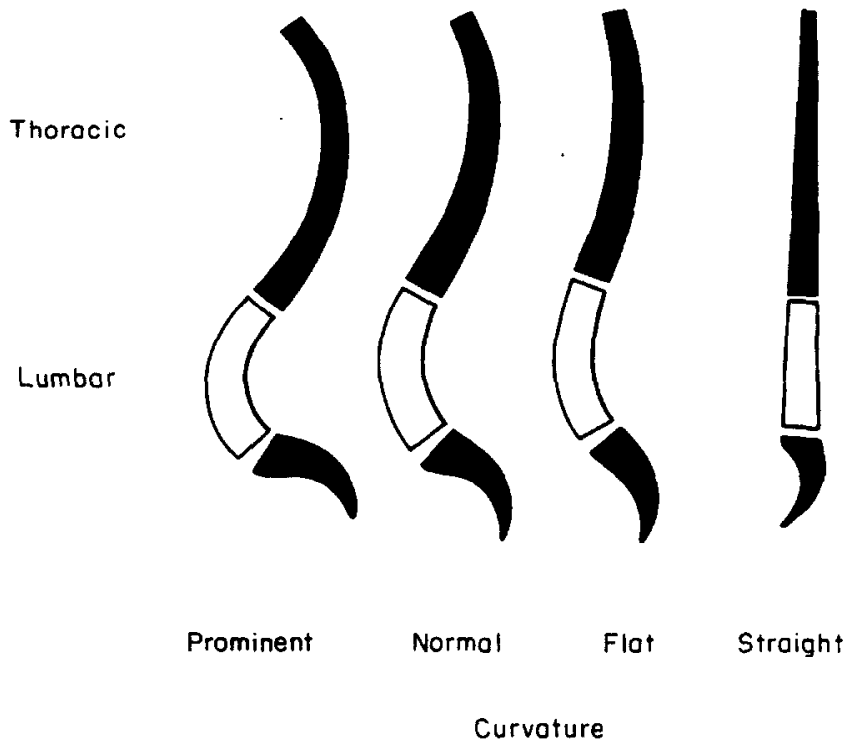

Figure 4. Various shapes of the thoracic and lumbar spine.

\section{The mechanical stability of the human spine}

There is a mechanically stable behaviour of the spine for loads smaller than the initial buckling load. However, no information can be obtained from the buckling theory about the amount of mechanical stability of the spine in the loading range from zero to the initial buckling load. In addition, in this range of loading there might be a large deflection of the spine, so that linear buckling theory no longer applies. In order to investigate the mechanical stability of the spine for large deflections, the current stiffness method is used. The behaviour of a nonlinear three-dimensional struc-

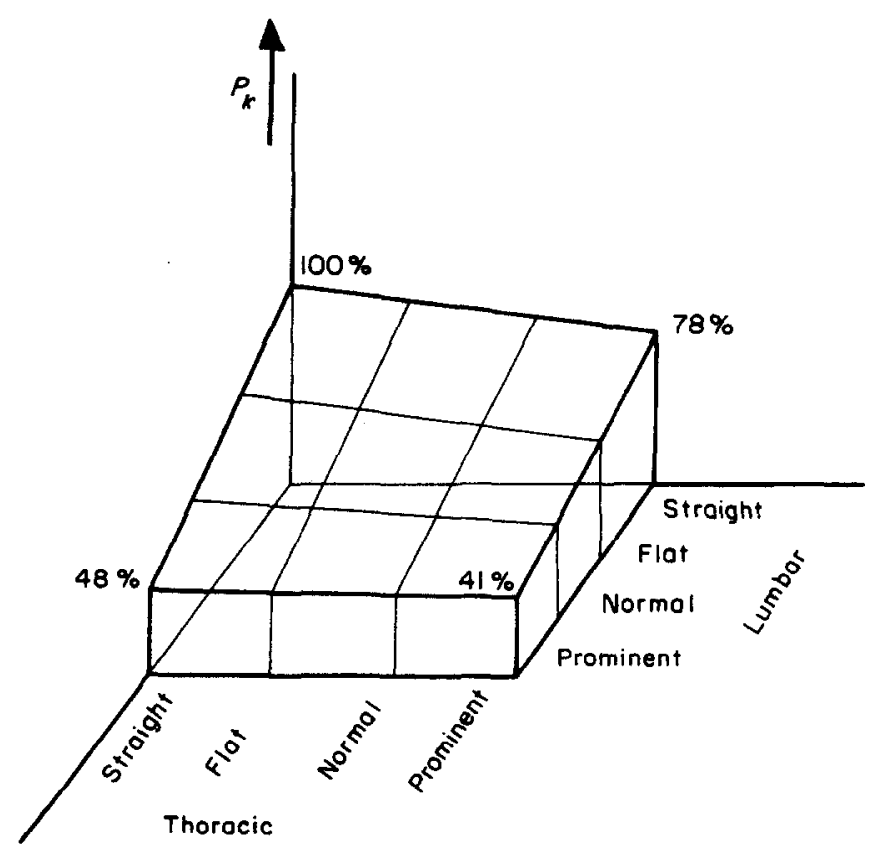

Figure 5. The initial buckling load with respect to the various shapes of the spine. ture can be characterized by a single value called 'the current stiffness parameter'. This method is described by Bergan et al. ${ }^{14}$. The incremental load vector $\Delta p_{i}$ during step number $i$ produces an incremental displacement $\Delta u_{i}$. A measure of the current stiffness of the structure may be expressed in terms of the inverse quantity of the normalized work. It follows:

$$
\mathrm{SP}_{\mathrm{i}}^{*}=\frac{\left|\Delta \mathrm{p}_{\mathrm{i}}\right|^{2}}{\Delta \mathrm{p}_{\mathrm{i}}^{\mathrm{T}} \Delta \mathrm{u}_{\mathrm{i}}}
$$

By means of the normalized load and displacement vector the parameter $\mathrm{SP}_{\mathrm{i}}^{*}$ is independent of the actual size of $\Delta \mathrm{p}$. By expressing the value SP ${ }_{i}^{*}$ with respect to its value at the first linear step, the current stiffness parameter $\mathrm{SP}_{\mathrm{i}}$ becomes:

$$
\mathrm{SP}_{\mathrm{i}}=\frac{\mathrm{SP}_{\mathrm{i}}^{*}}{\mathrm{SP}_{\mathrm{i}}^{*}}=\frac{\Delta \mathrm{p}_{\mathrm{i}}^{\mathrm{T}}}{\Delta \mathrm{p}_{\mathrm{i}}^{\mathrm{T}}} \cdot \frac{\Delta \mathrm{u}_{\mathrm{i}}}{\Delta \mathbf{u}_{\mathrm{i}}} \cdot \frac{\left|\Delta \mathrm{p}_{\mathrm{i}}\right|^{2}}{\left|\Delta \mathrm{p}_{\mathrm{i}}\right|^{2}}
$$

After the first step the current stiffness parameter SP equals 1 . For softening systems SP decreases and for stiffening systems SP increases. The current stiffness parameter has to become zero for mechanical instability.

\section{Influence of the curvature of the spine with respect to its mechanical stability}

In order to analyse the influence of the curvature of the spine on its mechanical stability, the current stiffness parameter was calculated. A normal physiological curved spine was analysed and compared to a straight spine. The geometrical nonlinear behaviour of the spine was analysed by using an incremental solution procedure (Zienkiwicz ${ }^{15}$ ). The load vector $\mathrm{P}$ for the weight of the upper part of the body was constructed out of several forces, each corresponding to the weight of a small slice of the body parallel to the transverse plane and acting at the slice centroid. The total amount of applied body weight was $380 \mathrm{~N}$. The incremental load vector was increased from zero up to twice the body weight. During every step the current stiffness parameter $\mathrm{SP}_{\mathrm{i}}$ was calculated.

In Figure 6a the behaviour of the current stiffness parameter as a function of the load is given. In the lower part of this figure, the behaviour of the current stiffness parameter as a function of the maximal horizontal deflection is shown. For a small deflection of the spine the current stiffness parameter equals almost 1 . By increasing the load vector the current stiffness parameter decreases. This means that the spine becomes less stable. However, after a load of 1.6 times body weight the current stiffness parameter again increases. This corresponds to a deflection of the top of the spine of about $200 \mathrm{~mm}$. There is mechanical instability if the current stiffness parameter is equal to zero. As shown in Figure 6 there is no instability of the spine for these conditions. If we suppose the same physical properties for the normal curved spine and the straight spine, it follows that the lordotic and kyphotic curvatures of the spine have a minor positive effect with 

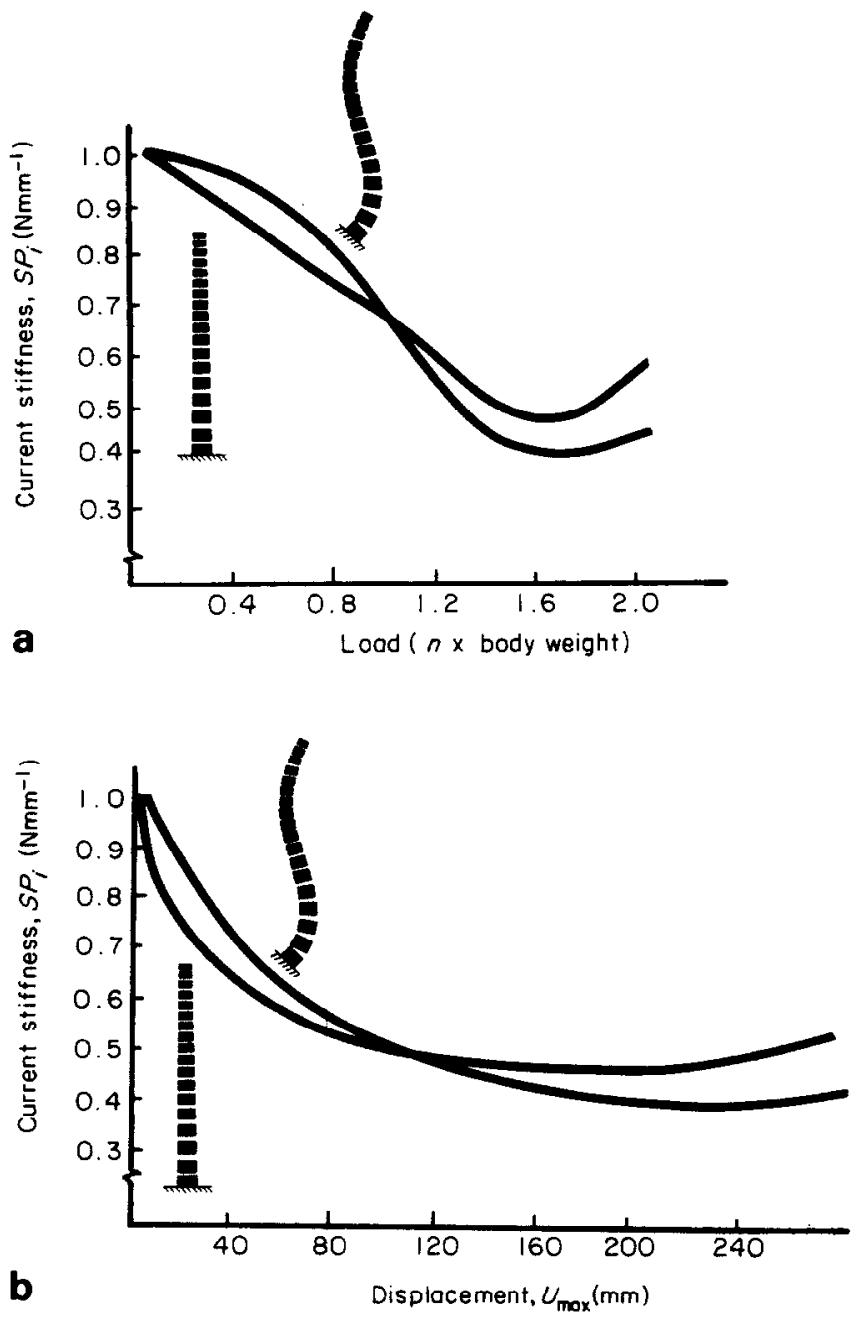

Figure 6. The behaviour of the current stiffness parameter as (a) a function of the load and (b) a function of the maximal horizontal deflection.

respect to the stability of the spine for a load smaller than or equal to the body weight.

\section{Influence of the physical properties of the spine with respect to its mechanical stability}

In order to analyse the influence of the physical properties of the spine on its mechanical stability, the bending stiffness of the spine has been changed. The bending stiffness of the spine depends on geometrical and physical properties of the interconnecting structures. The geometrical properties were determined by means of X-rays of a normal spine. The physical properties of the interconnecting structures are expressed by means of E, the Young's modulus of elasticity. In this analysis it was supposed that the Young's modulus was equal for all the intervertebral discs of the spine. The value of the Young's modulus $E$ of the intervertebral discs has been changed in such a way that the spine can bear its own weight in an upright position until a position of maximal flexion is reached. The load vector was constructed out of several forces to account

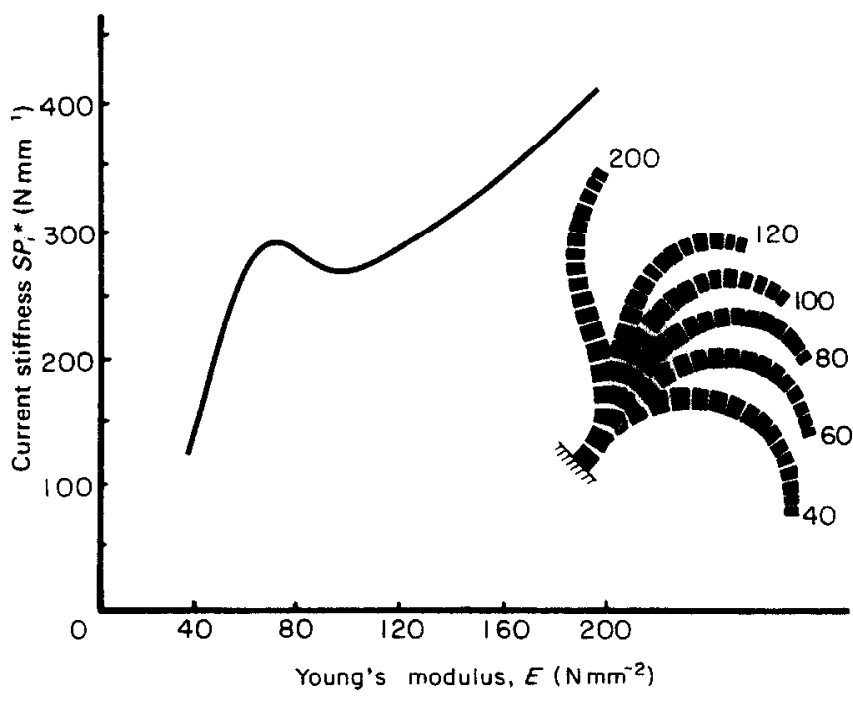

Figure 7. The current stiffness parameter as a function of the Young's modulus of elasticity $E$.

for the distribution of the weight of the body with a total amount of weight of $380 \mathrm{~N}$. After this, the Young's modulus $E$ was decreased in small steps. In every step the load vector for the weight of the upper part of the body was prescribed in increments to account for the geometrical nonlinear behaviour. During the last load increment the current stiffness parameter $\mathrm{Sp}_{i}^{*}$ was calculated. This process was repeated for the various values of the Young's modulus $E$. The corresponding positions of the spine in the loaded situations are shown in Figure 7. A value of $E=40 \mathrm{~N} /$ $\mathrm{mm}^{2}$ corresponds to a maximally flexed position of the spine.

If we calculate the current stiffness parameter for the various positions of the spine, by changing the Young's modulus $E$ of the intervertebral discs, Figure 7 can be constructed. The spine attains an unstable position for a current stiffness $\mathrm{Sp}_{i}^{*}$ value equal to zero. From this figure it follows that the spine behaves as a stable structure for the calculated situations. The local maximum corresponds to a change of the compression force into a tension force.

\section{Torsion instability}

Because of the lordotic and kyphotic curvatures of the spine there is not only a compression force but also. a shear force acting on the spine. A shear force acting on the spine may result in a torsion of the spine if the shear force does not act at the centre of transverse forces. This may result in torsion instability, which depends on the relation between the bending stiffness $E I$ and the torsion stiffness S. Applying the linear elastic theory for slender straight rods, the critical load $P_{1}$ for torsion instability equals:

$$
P_{1}=\frac{4 \sqrt{E I S}}{I^{2}}\left(\text { Timoshenko }{ }^{\prime \prime \prime}\right)
$$


If we compare this equation with the Euler formula for buckling:

$$
\mathrm{P}_{\mathrm{k}}=\frac{\pi^{2} \mathrm{EI}}{4 \mathrm{I}^{2}}
$$

torsion instability occurs if $\mathrm{S}<\frac{\pi^{4} \mathrm{EI}}{16^{2}} \simeq 0.38 \mathrm{EI}$

The torsion stiffness $S$ of the lumbar human ligamentous spine is more than twice the bending stiffness EI (Schultz et al. ${ }^{16}$ ). This makes torsion instability less likely.

\section{Discussion}

Lucas and $\mathrm{Bresler}^{7}$ have calculated the buckling load of an isolated spine and found a value of $20.9 \mathrm{~N}$. However, in vivo the trunk is capable of supporting much larger loads. By taking into account the body weight distribution along the spine, the calculated initial buckling load will be twice the value for the case of a single concentrated load. Scholten et al. ${ }^{17}$ have found that the bending stiffness of the whole trunk in vivo is about 10 times the bending stiffness of an isolated spine. This means that the initial buckling load for a whole trunk in vivo is at least $400 \mathrm{~N}$.

According to the linear buckling theory, the initial buckling load will decrease by increasing the curvature of the spine. Fick ${ }^{6}$ and later also Kapandji ${ }^{18}$ reported that the stability of the spine increases linearly with the square of the number of bends. This means that a straight spine is less stable than a curved spine. This is in contrast to the findings reported in this work.

Because the bending stiffness of the spine against lateral bending is of the same order as the bending stiffness against flexion, it follows that in the frontal plane the same relation between the initial buckling load and the curvature may be expected as in the sagittal plane. Since a normal spine is curved in the sagittal plane and straight in the frontal plane, it may be concluded that there is a higher incidence of buckling of the spine in the sagittal plane than in a frontal plane. Scoliosis is a lateral deviation of the spine in the frontal plane. This means that the initial buckling behaviour of a spine does not correspond to the deformity in scoliosis.

In the case of a slender rod, the buckling load is inversely proportional to the square of the length of the rod. If buckling is involved in scoliosis, the length of the spine is an important parameter. Skogland and Miller ${ }^{19}$ have found no significant difference as regards the lengths of the thoracolumbar spines between children with idiopathic scoliosis and controls. However, they found a higher index height/width for the thoracic as well as for the lumbar vertebral bodies in scoliotics. This indicates an increased slenderness of the spine in children with idiopathic scoliosis. Mattson et al. ${ }^{9}$ have found that girls with scoliosis had the same flexibility or were less flexible than normal girls. No difference was found in the bending stiffness during flexion of the spine between children with idiopathic scoliosis and controls by Veldhuizen and Scholten ${ }^{20}$. These findings suggest that spine flexibility has no dominant role in the progression of scoliosis. Greater spinal mobility and increased slenderness of the spine can be simulated by decreasing the bending stiffness of the spine model. Applying the large deflection theory, the results reported earlier show that the spine model behaves as a stable structure, even after decreasing the bending stiffness of the spine.

Bunch and coworkers ${ }^{21}$ have given a biomechanical explanation as to why larger scoliotic curves are more progressive than smaller curves. This was explained by means of the linear buckling theory. According to the results given in Figure 5, an increase of the curvature of the spine leads to a decrease of the initial buckling load. This means a higher incidence of buckling in a large scoliotic curve than in a small curve. On the basis of this, it may be concluded that larger scoliotic curves are more progressive than smaller curves, which is in agreement with the findings of Bunch et al. ${ }^{21}$ However, applying the large deflection theory, the results given in Figure 6 show that the human spine behaves as a stable system. Loading the spine with the calculated initial buckling load will result in a large deflection, so that the linear buckling theory no longer applies.

This means that it is incorrect to predict the progression of scoliotic spines based on the linear buckling theory. Why larger scoliotic curves are more progressive than smaller curves can possibly be explained by means of the lateral offset of the apex of a scoliotic spine. If there is a lateral offset of the apical vertebra, then there is a moment produced by the body weight acting at the spine. This moment may increase the scoliotic curve and results in a bigger lateral offset, which in turn, results in an increase of the moment.

In this study the intervertebral discs were described by means of beam elements. With these elements no relation between bending and torsion is taken into account. From structural engineering in systems with a coupled behaviour between bending and torsion we know that in this case the critical load can be much smaller than in the case of pure bending or torsion instability. If analysis of mechanical instability can help us in the understanding of the mechanism of scoliosis, more attention has to be paid to the coupling behaviour between bending and torsion. The rib cage contributes to the stability of the in vivo spinal column, but to an unknown degree (Andriacchi et al. ${ }^{22}$ ). Theoretically the rib cage is a system that can prevent buckling of the spine. However, this is not what occurs in practice, because in scoliosis the curves are most common at the thoracic level ${ }^{23}$. An understanding of the mechanics of the rib cage is important in the treatment of scoliosis. So, the role the rib cage plays in stabilising the human spine needs to be explored in depth.

It has been said that loading of the vertebrae by muscles and ligaments stimulates growth ${ }^{24}$. Both clinical and basic science studies are needed for the effect of the change of the geometrical properties (growth) of the spine in relation to its stability. 


\section{References}

1 Pope MH. Stokes IA. Moreland M. The biomechanics of scoliosis. Crit Rev Biomed Eng 1984; 157-88.

2 Nachemson AL. Sahlstrand T. Etiologic factors in adolescent idiopathic scoliosis. Spine 1977: 2: 176

3 White AA. Panjabi MM. The clinical biomechanics of the spine. Philadelphia: Lippincott 1978

4 Carcy EJ. Scoliosis-ctiology. pathogenesis and prevention of experimental rotary lateral curvature of the spine. JAMA 1932: 2: 104-10

5 Engel WK, Bean SC. Askanas V. The many causes of "idiopathic" scoliosis. Lancet 1975: 1: 228

6 Fick R. Handbuch der Anatomie und Mechanik der Gelenke, III Teil. Jena: Gustav Fischer 1911

7 Lucas DB. Bresler B. Stability of the ligamentous spine. (Technical report 40). San Francisco: Biomechanics Laboratory 1961

8 Haderspeck K. Schultz AB. Progression of idiopathic scoliosis. An analysis of muscles actions and body posture weight influences. Spine 1981:6:447-55

9 Matson G, Haderspeck K. Schultz A. Nachemson A. Joint flexibilities in structurally normal girls and girls with idiopathic scoliosis. J Orthop Res. 1983: 1: 57-62

10) Timoshenko S, Gene J. Theory of elastic stability. McGraw-Hill 1968

11 Scholten PJM. Idiopathic scoliosis: some fundamental aspects of the mechanical behaviour of the human spine Thesis. Amsterdam. The Netherlands 1986

12 Schultz AB. Belytschko T. Anolkiacchi T. Analog studies of forces in the human spine: Mech. properties and motion segmental behaviour. J Biomech 1973: 6: 373-8.3

1.3 Belytschko T. Andriacchi T. Schultz AB. Galante J.
Analog studies of forces in the human spine: computational techniques. J Biomech 1973: 6: 361-71

1+ Bergan PG. Soreide TH. Solution of large displacements and instability problems using the current stiffness parameter. Proceedings of finite elements in non-linear mechanics. Vol. 2. 1978.

15 Zienkiwicz OC. The finite element method. New York: McGraw Hill. 1977

16 Schultz AB. Warwick DN. Berkson MH. Nachemson AL. Mechanical properties of human lumbar spine motion segments, part I: responses in flexion. extension. lateral bending and torsion. J Biomed Eng 1979: 101: 46-52

17 Scholten PJM. Veldhuizen AG. The hending stiffness of the trunk. Spine 1986:11:46.37

is Kapandji IA. The physiology of the joints, Vol. III. Edinburgh: Churchill Livingstone 1972

19 Skogland LB. Miller JA. The length of the thoracolumbar spine in children with idiopathic scoliosis. Acta Orthop Scind 1981: 52: 77-185

2) Veldhuizen AG. Scholten PJM. Flexibility in structurally normal girls and girls with idiopathic scoliosis (submitted)

21 Bunch WH. Patwardhan AG. Vanderby R. Stability of scoliotic spines. Proceedings I ith Annual Meeting of Scoliotic Research Society, 1983

22 Andrialcchi T. Schultz AB. A model for studies of mechanical interactions between the human spine and rib calge. J Biomech 1974: 7: 497-5(17)

23 Brooks HL. Azen SP. Gerberg E. Brooks R. Chan L. Scoliosis: a prospective epidemiological study, J Bone Joint Surg 1975: 57A: 968-72

2f Arkin AM. Katz JF. The effect of pressure on epiphyseal growth. The mechanism of plasticity of growing bone, J Bone Joint Surg 1956: 38A: 1056-6.5 